\title{
EDGE BASED ECOSYSTEM FOR INTERNET OF THINGS
} (EBEFIOT)

\author{
S Lokehwar, A Hemaranjanee, V. Narayanee
}

\begin{abstract}
This paper describes about an entirely new ecosystem which is designed for the future to provide resources to IOT devices. This is a hybrid ecosystem which combines the edge computing capabilities along with a new functionality that aims to provide the end users to benefit more accurate and faster experience compared to existing solutions. Apart from proving processing power and storage (like edge computing), the key advantage of this ecosystem is to provide more locality specific information to the end devices. It also utilizes the $5 G$ infrastructure. This paper provides an insight of various aspects of this ecosystem like its functionalities, use cases, implementation, etc.
\end{abstract}

Keywords - EBEIOT-IOT- cloud-edge-MDC-MEC-plugin- latency- bandwidth-server- ULI-AIdeployment- base station.

\section{Reference to this paper should be made as follows:}

$S$ Lokewar, A Hemaranjanee and V. Narayanee (2021), "Edge Based Ecosystem For Internet Of Things (EBEFIOT)" Int. J. of Electronics Engineering and Applications, Vol. 9, No. 1, pp. 58-67, doi 10.30696/IJEEA.IX.I.2021.58-67.

\section{Biographical notes:}

S Lokehwar : Computer Science and Engineering from PERI Institute of Technology,.

A Hemaranjanee : Computer Science and Engineering from PERI Institute of Technology,

V. Narayanee : Computer Science and Engineering from PERI Institute of Technology,. 


\section{[1] INTRODUCTION}

It is evident that the near future is expected to be filled with enormous number of IOT devices in all possible fields. Most of these devices require resources from an external source (like a server or cloud). EBEFIOT is an additional module that enhances the resource querying and processing functionalities of an IOT device. EBEFIOT provides all those resources and processing power along with Unique Location Information (ULI). This can be used in any devices which are connected to the internet, ranging from small IOT devices to full sized computers. This ecosystem enables any device in the internet with the EBEFIOT plugin to access the ecosystem. The server provides the data which is more detailed and specific to that particular locality. For example, Chennai has many localities, the weather (temperature, rainfall, etc.) differs in each locality. Although, if you ask your virtual assistant, it just gives average weather information which in most of cases differs from the actual weather. EBEFIOT solves this problem as the server consists of the exact weather information for each locality. EBEFIOT is also capable of providing storage and computation similar to an edge computing implementation. This is just one simple example. There are numerous uses and applications for this ecosystem that could enhance the speed, accuracy, processing power and the computation power of the future IOT and other connected devices[1-4]

\section{[2] EDGE COMPUTING}

Edge computing is a modified implementation of cloud computing. Cloud computing is the delivery of different services through the Internet. These resources include tools and applications like data storage, servers, databases, networking, and software. Rather than keeping files on a proprietary hard drive or local storage device, cloud-based storage makes it possible to save them to a remote database. As long as an electronic device has access to the web, it has access to the data and the software programs to run it [5-7].

Edge computing is a networking philosophy focused on bringing computing as close to the source of data as possible in order to reduce latency and bandwidth use. In simpler terms, edge computing means running fewer processes in the cloud and moving those processes to local places, such as an edge server. Bringing computation to the network's edge minimizes the amount of long-distance communication that has to happen between a client and server [8-12].

In order to implement EBEFIOT, we are using Multi-Access Edge Computing technique. MultiAccess Edge Computing (MEC) moves the computing of traffic and services from a centralized cloud to the edge of the network and closer to the customer. Instead of sending all data to a cloud for processing, the network edge analyses, processes, and stores the data. Collecting and processing data closer to the customer reduces latency and brings real-time performance to high-bandwidth applications.

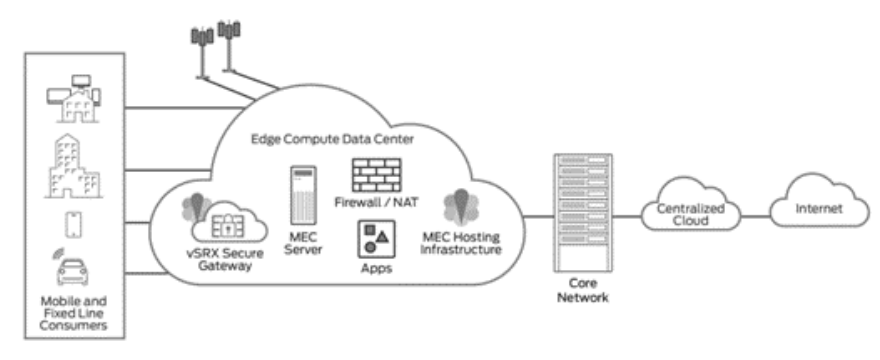

Fig. 1. Multi Access Edge Structure 


\section{[3] ADVANTAGES OF USING MEC}

This type of implementation has many advantages.

-Super Low Latency: The computing source is present closer to the user device, which results in super low latency. The latency gets reduced from a few hundred milliseconds to single digit numbers. -Reduced Bandwidth: Next major advantage is that, since all the resources need not be taken to the cloud, it drastically reduces the bandwidth requirement.

-Storage and Computational Power: All the devices can be aided with storage and computational power. It enables Real-time AI data analysis and enhanced application performance.

-Lower operating costs: This system results in lower operating cost as the distance and the amount of data to be transmitted is drastically reduced.

-Reduced Downtime: Since all the devices does not depend on a single centralized device, even if one device is down that does not affect all the end devices. Also, the size of the edge server is smaller when compared to the cloud, thus less maintenance time is required and the server downtime is considerably reduced.

-Enables New Services and Revenue Streams: This has the potential to enable a plethora of new services in the future,

-Multipurpose Infrastructure: The infrastructure required for edge computing can be used for 5G networks and vice versa.

-Real-time access to radio network information that can be leveraged by applications.

\section{[4] WHAT IS EBEFIOT?}

EBIOT is an ecosystem, which assists all the devices connected to internet.

It mainly aims to serve the following:

1.It enables any device connected to the internet to use this ecosystem (ranging from google AI,Alexa, Siri, etc. to a DIY setup made by a student).

2.Provide more detailed information regarding the user device's locality will be loaded into the MDCs like the weather condition of the particular area, detailed map info, landmarks, addresses, etc. 3.Provide resources such as processing power and storage in the edge.

4.Consists of an AI engine built into the MDC to effectively serve the end devices.

5.Reduce the latency and bandwidth requirements when compared to existing cloudbasedprocessing.

EBEFIOT has a Micro Data Centre (MDC) at the server side and a plugin in the client side. MDCs are placed along with the user device's respective base station. Any device in this ecosystem will communicate to the MDC using this plugin.

It mainly aims to provide localized information whenever necessary. It results in providing more accurate resources with less latency. It also maintains a log of the activities carried out in that locality which enables effective integration of services in the locality if required. Since most of the processing is done in the MDC itself, it eliminates the need for cloud for all kinds of processing requirements, thus reducing the traffic at the cloud and reduces bandwidth. It is evident that IOT 5G is booming rapidly, the number of devices will be tripled over the next decade. But using EBEFIOT makes it easier to manage the large number of devices and keeping them up and running with higher accuracy and low latency. On the larger scale, using this system reduces the processing energy required per 
TB of data. This is achieved by reducing the bandwidth requirements. Bandwidth is reduced as physical distance is reduced by at least 50 times in most of the cases.

\section{[5] EBEFIOT - USE CASES AND BENEFITS}

Let us see some of the application and benefits of EBEFIOT.

5. A) Let us consider a use case that we use on a daily basis. We all have weather information for our location in our devices. But that information is usually the average information of the overall city. It turns out to be inaccurate most of the times. Using EBEFIOT this problem can be solved by: If you are asking to your AI assistant/ looking the weather widget, the system program asks the EBEFIOT plugin for the weather information. The plugin communicates with the MDC for the information. The weather information for the corresponding localities are stored in MDC. Thus, the accurate weather information is returned to the user. In MDC the weather information is updated at regular time intervals by communicating with the forecast center which also will be connected to the internet. If the user asks for the information about some other location, then it will be linked to the corresponding MDC to provide that information.

BENEFIT: This kind of detailed information is only stored in the localized MDC and not in the cloud. The cloud consists only the information of that city as usual. It is unnecessary to store that much detail in a cloud. Since all the details are stored and the request is processed in the EBEFIOT MDC itself, both the latency and bandwidth are reduced and at the same time, more accurate information is provided to the end user at very less time compared to the conventional method. Also, any device/ software can make use of this environment as all the devices have access to this ecosystem.

Since this ecosystem is mainly aimed to assist the future, let us see some of the futuristic use cases

5. B) We have started implementing robots in all the possible places. In future, robots maybe used to clean roads and drainage systems. To perform this task, the robots need a detailed map of the road (unlike google maps they require the street details and precise routes) and drainage system of the proximity in which they are deployed. They may also require the information regarding the places which are already cleaned by the other robot. Now, maintaining the entire drainage system's details in a remote cloud server at some other Country is inappropriate. Thus, all these detailed information are stored in the MDCs of that particular locality. Now the robots can use this information to do their work with more precise inputs. Also, the real time data such as traffic, road block, etc. can be transmitted to the robots with very less latency.

BENEFIT: Unnecessary resources are eliminated from the cloud. The end device is provided with more detailed information with minimum bandwidth usage. Any real-time data can be sent with minimum delay.

5. C) Another use case can be drones. Drones are to be deployed for the delivery of various things to households. A drone delivers things based on the location but it requires a lot of details and remote processing. This service also requires active location tracking. This can be done by deploying Geo location tracking software in the MDCs. Details that will make easier for the drone and make it more 


\section{S Lokehwaret al.}

accurate can be placed in the EBEFIOT MDCs. This minimizes the latency and also enables better accuracy of the drone.

-This system facilitates most of the cloud-based services which are currently available only to the business users, also accessible by domestic users.

-Another 'thing' of the future is smart cities. Smart cities would have many connected devices and involve a lot of management activities. Carrying out all those activities in a central location may result in increased bandwidth requirement, latency and increases the complexity of management. Thus, carrying out activities at the edge itself and only transmitting the required information to the centralized place results in a more efficiency.

-Another and one of the most underrated features of this edge / cloud-based system is the reduction in the carbon footprint. As resources are processed in a common location, the need for a powerful computational system at the user level is eliminated, thus reducing the carbon footprint of each of the device.

\section{[6] STRUCTURE OF EBEFIOT}

The basic infrastructure required for EBEFIOT can be utilized from Multi-Access Edge Computing infrastructure. The standards for deploying Multi Access Edge computing networks (MEC) are defined by European Telecommunications Standards Institute (ETSI).

According to ETSI, the deployment standards for MEC servers (which constitute the basic structural units for the deployment of EBEFIOT MDCs) are:

The MEC application server can be deployed at the macro base station EnodeB that is part of an LTE cellular network, or at the Radio Network Controller (RNC) that is part of a 3G cellular network and at a multi-technology cell aggregation site. The multi-technology cell aggregation site can be located indoors or outdoors.

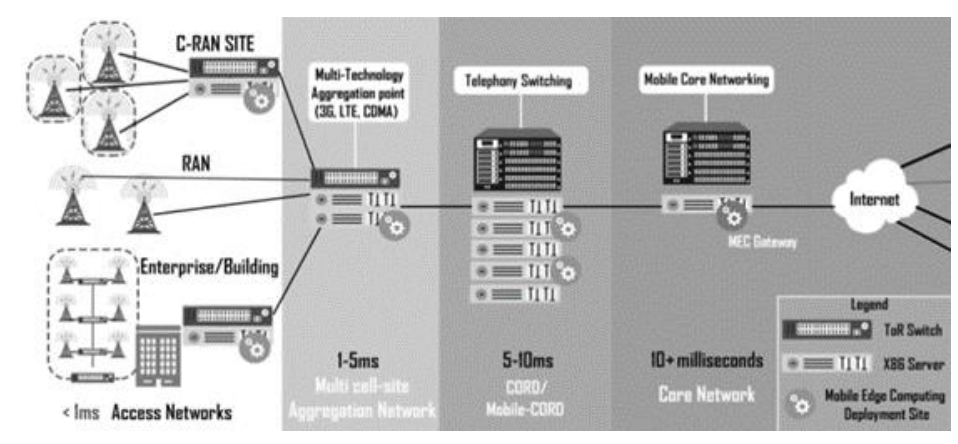

Fig. 2. Multi Access Edge Network

If EBEFIOT ecosystem has to be setup along with already existing $3 \mathrm{G}$ or $4 \mathrm{G}$ network, then the deployment of the MEC servers can be done at 3 places:

-For deploying the servers in already existing LTE network: The servers / the Micro Data Centers can be deployed at the LTE macro base station. 


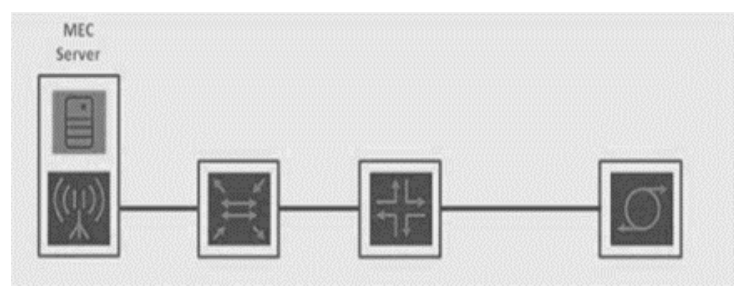

Fig. 3. MEC at the LTE macro base station (eNB)site

- The MEC server can also be deployed at the multi-technology $3 \mathrm{G}$ cells aggregation site, if it has to deployed on $3 \mathrm{G}$ network or can also be deployed at the multi-technology 4G LTE cells aggregation site, if it has to deployed on $4 \mathrm{G}$ network.

-The multi-technology (LTE/3G) cell aggregation site can be located indoor within an enterprise (e.g. hospital, large corporate HQ), or Indoor/outdoor for a special public coverage scenario (e.g. stadium, shopping mall) to control a number of local multi-technology (3G/LTE) access points providing radio coverage to the premises. This deployment option enables the direct delivery of locally-relevant, fast services from base station clusters.

-MEC servers can also be deployed at the Radio Network Controller (RNC) site.

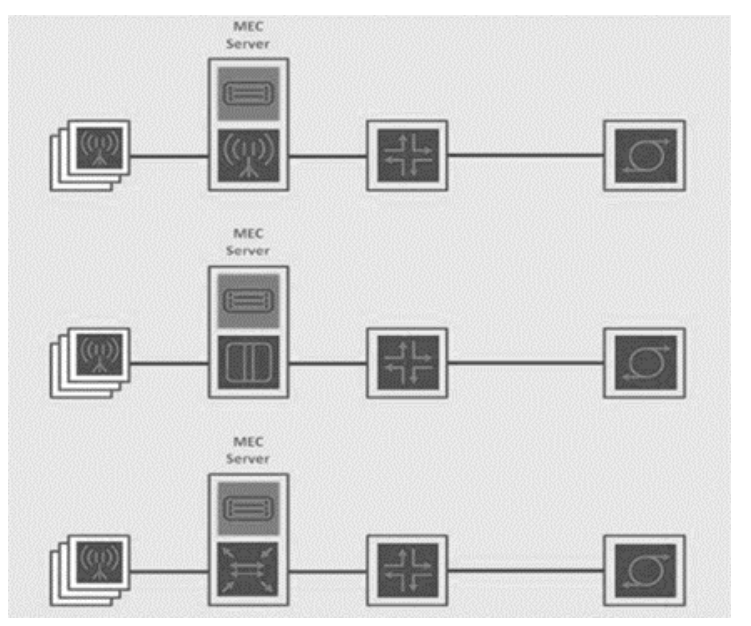

Fig. 4. MEC at multi-technology (3G/LTE) cells aggregation site

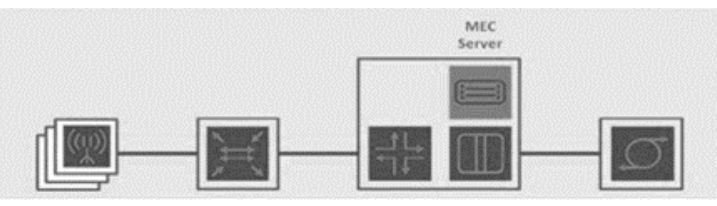

Fig. 5. MEC at Radio Network Controller (RNC) site.

The EBEFIOT MDCs (Micro Data Centers) are basically a combination of many servers. They are deployed in the Macro Base Stations of the LTE/5G network.

The EBEFIOT MDCs can be possibly placed at 2 different places:

1.Directly along with the base station.

2.Can be placed at a minimum distance from the base station and can be connected to it.

If the service provided by the ecosystem is to be utilized irrespective of the network service providers, then it has to be placed in a common location nearby the base stations of all the network providers, 
and the MDC has to be connected to all the base stations. But this kind of implementation will increase the latency a little bit and also demands for more resource management / processing system. Thus, to get maximum efficiency, the MDC should be placed along with the base station.

\section{[7] COMPONENTS AND WORKING OF EBEFIOT}

EBEFIOT constitutes two basic components:

1.EBEFIOT Micro Data Centre (MDC).

2.EBEFIOT Plugin.

\section{A. EBEFIOT Plugin}

The communication to the MDC can be done using this plugin.

- This plugin can be made available to the users by making it available online.

- This is a light-weight plugin which is capable of auto configuring itself according to the target device.

- This plugin can be attached with the UI of the user's end device.

- Any AI/software program communicates with the plugin, it establishes a tunnelled connection between the MDC and the plugin.

- This plugin supports all the services of the ecosystem and facilities to provide all the feasible requests from the interface.

- EBEFIOT plugin utilizes the API (Application Programming Interface) to enable all the functions.

\section{B. EBEFIOT Micro Data Centre (MDC)}

This Micro Data Centre is responsible for processing all the user requests, providing storage, storing Unique Location Information (ULI), running the remote programs and applications, etc.

Main Functionalities of MDC:

- Storing and providing ULI (Unique Location Information). This is the unique and distinctive feature of this ecosystem. It stores and provide optimized local content.

- Provides Software as a Service (SaaS). It enables the user's application to be run on EBEFIOT, thus reducing the need for the user having powerful processors and it also reduces the user's burden of maintenance operations as the application is operated and maintained by EBEFIOT.

- Providing virtualization capabilities to the devices (if necessary). Like any other cloudbased service, EBEFIOT is capable of providing storage and CPU processing as a service to the user's end devices.

- Provides all the services provides by any cloud platform with super low latency and less bandwidth.

- Provide Platform as a Service (PaaS).

- Provide Infrastructure as a Service (IaaS) i.e. provides business and domestic access to web infrastructure such as servers, connection, storage space, etc. 
There are four main components inside the MDC:

1. EBEFIOT AI Engine.

2. Virtualization Unit.

3. Processing Unit.

4. ULI (Unique Locality Information) Database.

5. General Database.

MDC receives the requests, commands, etc. and sends the response, data, and etc. from the plugin (in the end device) by means of the service provider's network. As soon as it receives an input, various components perform their respective tasks.

EBEFIOT AI Engine: This AI Engine receives the input from the plugin and it immediately processes the input and decides what kind of task to perform. It initiates the things required to accomplish the given input and sends the output if necessary.

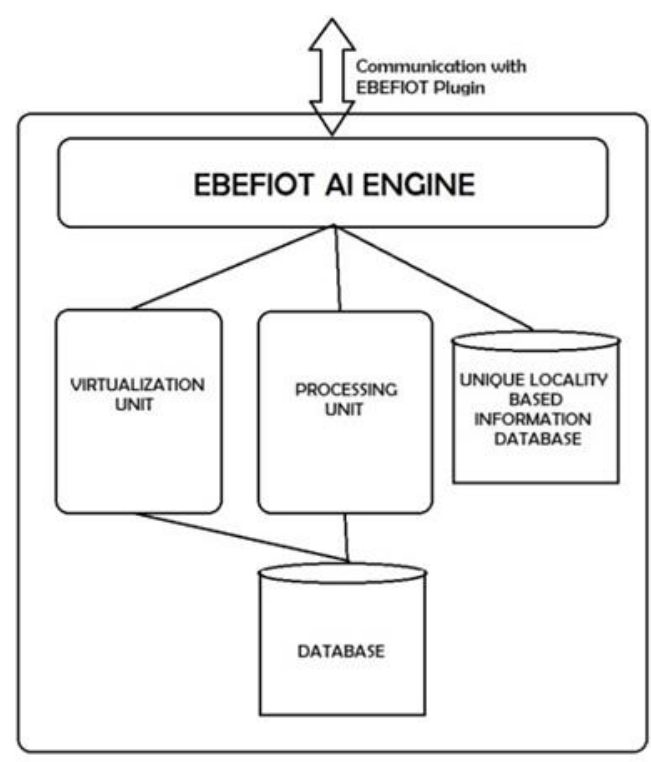

Fig. 6. Basic Architecture of EBEFIOT MDC

Virtualization Unit: It is responsible for providing Software as a Service (SaaS), Platform as a Service (PaaS), and also enables the end device to virtually run a program or application using the $\mathrm{CPU}$ and storage of the MDC rather than using that of the end device.

Processing Unit: This unit is the heart of the ecosystem. It performs all the processing required to accomplish the tasks given to the ecosystem. It consists of many powerful CPUs and GPUs depending on the requirement.

ULI (Unique Locality Information) Database: This is the component that enables the ecosystem to uniquely provide accurate information to the end users. This database is used to store all the data which is exclusive to that particular locality such as weather information, detailed maps, etc.

General Database: This is the typical database that stores all types of data other than ULI data. 


\section{S Lokehwaret al.}

\section{[8] USAGE RESULT}

If EBEFIOT is brought to use, some advantages apart from the obvious ones are:

- Improve mobile user's Quality of Experience (QoE), by reducing latency.

- Improving quality of service or/and providing customized services.

- Improve infrastructure's efficiency, with more intelligent and optimized networks.

- Enable disruptive vertical services, particularly relevant for, Big Data management, Analytics, Smart Cities and much more.

- Tight integration with radio equipment, making it easy to understand traffic characteristics and needs, deal with radio conditions, get device location information, etc. 


\section{REFERENCES}

[1] M. Navya, et.al,“Android based children tracking system using voice recognition”, International journal of Computer science and information technology, $\operatorname{Vol} 4$ (1)

[2] Abu Salim, Sachin Tripathi and Rajesh Kumar Tiwari “A secure and timestamp-based communication scheme for cloud environment" Published in International Journal of Electronic Security and Digital Forensics, Volume 6, Issue 4, 319-332.

[3] Rajesh Kumar Tiwari and G. Sahoo, "A Novel Watermark Scheme for Secure Relational Databases" Information Security Journal: A Global Perspective, Volume 22, Issue 3, July 2013.

[4] Md.Foisal Mahedi Hasan \& et al., "RFID-based Ticketing for Public Transport System: Perspective Megacity Dhaka, 3rd IEEE International Conference on Computer Science and Information Technology (ICCSIT), vol. 6, pp. 459-462, 2010.

[5] Varun Krishna K.G., Selvarathinam S., Roopsai V., Ram Kumar R.M., "Modified Ticketing System using Radio Frequency Identification (RFID)," International Journal of Advanced Computer Research, vol. 3, Issue 12, pp. 92-98, 2013.

[6] Arul Das, S.V.K.lingeswaran," GPS Based Automated Public Transport Fare Collection Systems Based on Distance Travelled By Passenger Using Smart Card.International Journal of Scientific Engineering and Research (IJSER), vol. 2, issue 3, March, 2014.

[7] Dalip, Vijay Kumar,"GPS and GSM based Passenger Tracking System”, International Journal of Computer Applications, vol. 100, no.2, August 2014.

[8] V. Ramesh and K. Ramar, 2011. "Classification of Agricultural Land Soils: A Data Mining Approach", Agricultural Journal, 6: 82-86.

[9] N.Hemageetha,"A Survey on application of datamining techniques to analyse the soil for agriculture purpose", INDIA Com, 2016.

[10] UnmairAyub, Syed AtifMosqurrab, "Prediction cross diseases using data mining approaches:Classification",ICPESG,2018.

[11] D.Ramesh, VishnuVaradhan, "Data Mining Techniques and Application to Agricultural Yield Data", IJARCCE, 2013.

[12] Anoop Joyti Sahoo, and Rajesh Kumar Tiwari “A Novel Approach for Hiding Secret data in Program Files" International Journal of Information and Computer Security. Volume 8 Issue 1, March 2016, 